Review Article

\title{
Disrupted Skeletal Muscle Mitochondrial Dynamics, Mitophagy, and Biogenesis during Cancer Cachexia: A Role for Inflammation
}

\author{
Brandon N. VanderVeen, Dennis K. Fix, and James A. Carson \\ Integrative Muscle Biology Laboratory, Department of Exercise Science, University of South Carolina, Columbia, SC, USA \\ Correspondence should be addressed to James A. Carson; carsonj@mailbox.sc.edu
}

Received 31 March 2017; Revised 6 June 2017; Accepted 19 June 2017; Published 13 July 2017

Academic Editor: Moh H. Malek

Copyright (c) 2017 Brandon N. VanderVeen et al. This is an open access article distributed under the Creative Commons Attribution License, which permits unrestricted use, distribution, and reproduction in any medium, provided the original work is properly cited.

\begin{abstract}
Chronic inflammation is a hallmark of cancer cachexia in both patients and preclinical models. Cachexia is prevalent in roughly $80 \%$ of cancer patients and accounts for up to $20 \%$ of all cancer-related deaths. Proinflammatory cytokines IL- 6 , TNF- $\alpha$, and TGF- $\beta$ have been widely examined for their regulation of cancer cachexia. An established characteristic of cachectic skeletal muscle is a disrupted capacity for oxidative metabolism, which is thought to contribute to cancer patient fatigue, diminished metabolic function, and muscle mass loss. This review's primary objective is to highlight emerging evidence linking cancerinduced inflammation to the dysfunctional regulation of mitochondrial dynamics, mitophagy, and biogenesis in cachectic muscle. The potential for either muscle inactivity or exercise to alter mitochondrial dysfunction during cancer cachexia will also be discussed.
\end{abstract}

\section{Introduction}

Pathological inflammation, a hallmark of numerous chronic diseases, can lead to fatal comorbidities, including cachexia [1-4]. Cachexia is characterized by unintentional body weight loss secondary to an underlying disease $[1,3]$ and is prevalent in $\sim 60-80 \%$ of cancer patients. Cancer patients exhibiting cachexia have increased fatigue, decreased functional independence, reduced life quality, and decreased survival [5-10]. Although no treatments are currently approved for cancer cachexia, improving the mechanistic understanding of skeletal muscle mass loss and more recently skeletal muscle metabolic function is thought to be central to the etiology of cancer cachexia and the successful development of therapeutic interventions.

Skeletal muscle mass and metabolism have established roles for maintaining health in obesity, ageing, and chronic disease [11-13]. Related to health, skeletal muscle serves as an amino acid reservoir for the body and a primary site of insulin-stimulated glucose transport [11, 14]. However, skeletal muscle relies heavily on lipids as a fuel source during rest and low-intensity activities and contributes to over $20 \%$ of whole body fatty acid metabolism [14]. This oxidative metabolism dependence underscores the muscle mitochondria's critical role in metabolic homeostasis $[14,15]$. The analysis of muscle oxidative metabolism involves the quantification of mitochondria content, respiratory capacity, and the efficiency of the Krebs cycle and electron transport chain (ETC) $[16,17]$. This line of inquiry has significantly advanced our mechanistic understanding of aging, disease, and physical inactivity's effects on muscle metabolism.

Dysfunctional muscle oxidative metabolism occurs with many disease conditions [12,13,18-21] and can involve mitochondrial dynamics, mitophagy, and biogenesis regulation $[18,22]$. Each of these dysfunctions is being actively investigated for their role in the pathogenesis of cancer cachexia $[12,13,23]$. Skeletal muscle mitochondrial dysfunction has been reported with cachexia in cancer patients and preclinical models $[12,15,24-27]$ and is consistent with functional changes involving increased muscle fatigability 
and overall weakness $[5,6,8,9,28]$. Accelerated catabolism and suppressed anabolism in wasting muscle has been linked to mitochondrial dysfunction $[12,25,26]$. The primary objective of this literature review is to highlight evidence linking cancer-induced inflammation to the regulation of muscle mitochondrial dynamics, mitophagy, and biogenesis. We will stress research areas that warrant further investigation to establish if they are a consequence of cachexia or a cause of the pathology. The examination of inflammatory mediators of cancer cachexia will be delimited to interleukin 6 (IL-6), tumor necrosis factor $\alpha$ (TNF- $\alpha$ ), and transforming growth factor $\beta$ (TGF- $\beta$ ) superfamilies' role. Evidence for these cytokines in the overall regulation of cachexia progression and muscle mass loss has been extensively reviewed elsewhere [29-38] and will only be briefly described here. We will also discuss the potential for either muscle inactivity or exercise to alter the regulation of dysfunctional mitochondrial dynamics, mitophagy, and biogenesis during cancer cachexia.

\section{Overview of Inflammation as a Driver of Cancer Cachexia}

2.1. Overview. Increased systemic inflammation is an established driver of cachexia development in numerous chronic diseases, including cancer [3]. Several cytokines have been implicated as the mediators of chronic inflammation for cachexia progression in both human and preclinical animal models [1, 29, 39]. Cytokines can regulate intracellular signaling that induces muscle wasting in response to various stimuli. IL-6, TNF- $\alpha$, and TGF- $\beta$ are cytokines that have been mechanistically linked to skeletal muscle wasting and disrupted metabolic homeostasis during cancer cachexia [29-38].

2.2. Interleukin-6. The IL-6 cytokine family has been widely investigated in skeletal muscle remodeling due to exercise, aging, and disease [29, 40-43]. IL-6 is a pleiotropic cytokine implicated as a critical regulator of inflammation-induced skeletal muscle and fat wasting during cancer cachexia [35]. Elevated circulating IL-6 can be observed in cachectic cancer patients and preclinical models alike and is strongly correlated to body weight and muscle mass loss [44-46]. IL-6 signals through the ubiquitously expressed glycoprotein 130 (gp130) receptor to activate downstream intracellular signaling pathways $[42,47,48]$. While IL- 6 can activate numerous cellular signaling pathways, the phosphorylation of immediate downstream target signal transducer and activator of transcription 3 (STAT3) has been most widely examined with cachexia-induced muscle mass loss [41, 49, 50]. STAT3 activation by IL- 6 causes the disruption of skeletal muscle proteostasis through both anabolic and catabolic signaling [51]. STAT3 inhibition can attenuate body weight and muscle mass loss in tumor-bearing mice $[52,53]$. This review will discuss the implications for IL-6-induced STAT3 signaling in the regulation of cachexia-induced mitochondrial dysfunction (Figure 1).

2.3. Tumor Necrosis Factor $\alpha$. TNF- $\alpha$ 's role in muscle wasting during cachexia has been well studied $[1,34,54-56]$. TNF- $\alpha$, released from activated macrophages, can activate skeletal muscle nuclear factor $\kappa \mathrm{B}(\mathrm{NF}-\kappa \mathrm{B})$ transcription factor and promote protein degradation through the transcription of ubiquitin proteasome E3 ligases, MurF1, and Atrogin1 $[34,38]$. Muscle MurF1 and Atrogin1 expression are prevalent in cancer patients and preclinical cachexia models and promote skeletal muscle protein degradation [57]. TNF- $\alpha$ can also promote body weight loss through the loss of adipose tissue by stimulating lipolysis and inhibiting lipogenesis [34]. However, TNF- $\alpha$ also promotes anorexia [58, 59]. Cancer-induced TNF- $\alpha$ levels increase corticotrophinreleasing hormone $(\mathrm{CRH})$, which reduces appetite and food intake $[33,34,60]$. However, TNF- $\alpha$ overexpression in mice lacking tumors induced weight loss, which was not different than pair-fed controls [61]. This portion of the review will focus on TNF- $\alpha$ 's induction of NF- $\kappa$ B to disrupt mitochondrial homeostasis (Figure 1).

2.4. Transforming Growth Factor $\beta$. TGF- $\beta$ cytokine super family consists of 34 proteins that regulate a myriad of cellular functions. Several family members have been found to promote cancer-induced skeletal muscle wasting [30, 62]. TGF- $\beta 1$, Activin A, TNF like weak inducer of apoptosis (TWEAK), and myostatin are TGF- $\beta$ super family members that bind to either type I or type II activin receptors in skeletal muscle and activate Smad (SMA, mothers against decapentaplegic) signaling $[1,30,56,63-66]$. Smad regulation of skeletal muscle wasting is still an area of active inquiry, but evidence suggests a role for forkhead box O3- (FOXO3-) dependent protein degradation as well as protein synthesis suppression through protein kinase B (Akt) [30, 38]. Activin $A$ administration can induce the cachectic phenotype in nontumor-bearing mice through $\mathrm{Smad} / 3$ activation, which increases atrophy and fibrotic gene transcription [67]. While TGF- $\beta$ signaling's role in cachexia continues to be elucidated, this review will discuss evidence for the TGF- $\beta$ superfamily to regulate skeletal muscle mitochondria function (Figure 1).

\section{Mitochondrial Dysfunction in Cachectic Muscle and Inflammatory Mediators}

3.1. Overview. Cachexia can be defined as a complex metabolic syndrome, and thus skeletal muscle mitochondria have become an intriguing focus for determining the underpinnings of cancer-induced muscle catabolism [3]. To this end, the maintenance of mitochondrial content and capacity for ATP production in cachectic muscle have become active areas of inquiry. While numerous studies have reported mitochondrial content loss with wasting, there remains a need to better define mitochondrial function and the regulators of this process in cachectic muscle. Mitochondrial function is classically defined as the capacity for ATP production through oxidative phosphorylation and betaoxidation $[24,68,69]$. Disruptions to the ETC decrease mitochondrial respiration and the ability to produce ATP. While relatively few published studies have directly examined muscle mitochondrial respiration with cancer cachexia, cachectic 


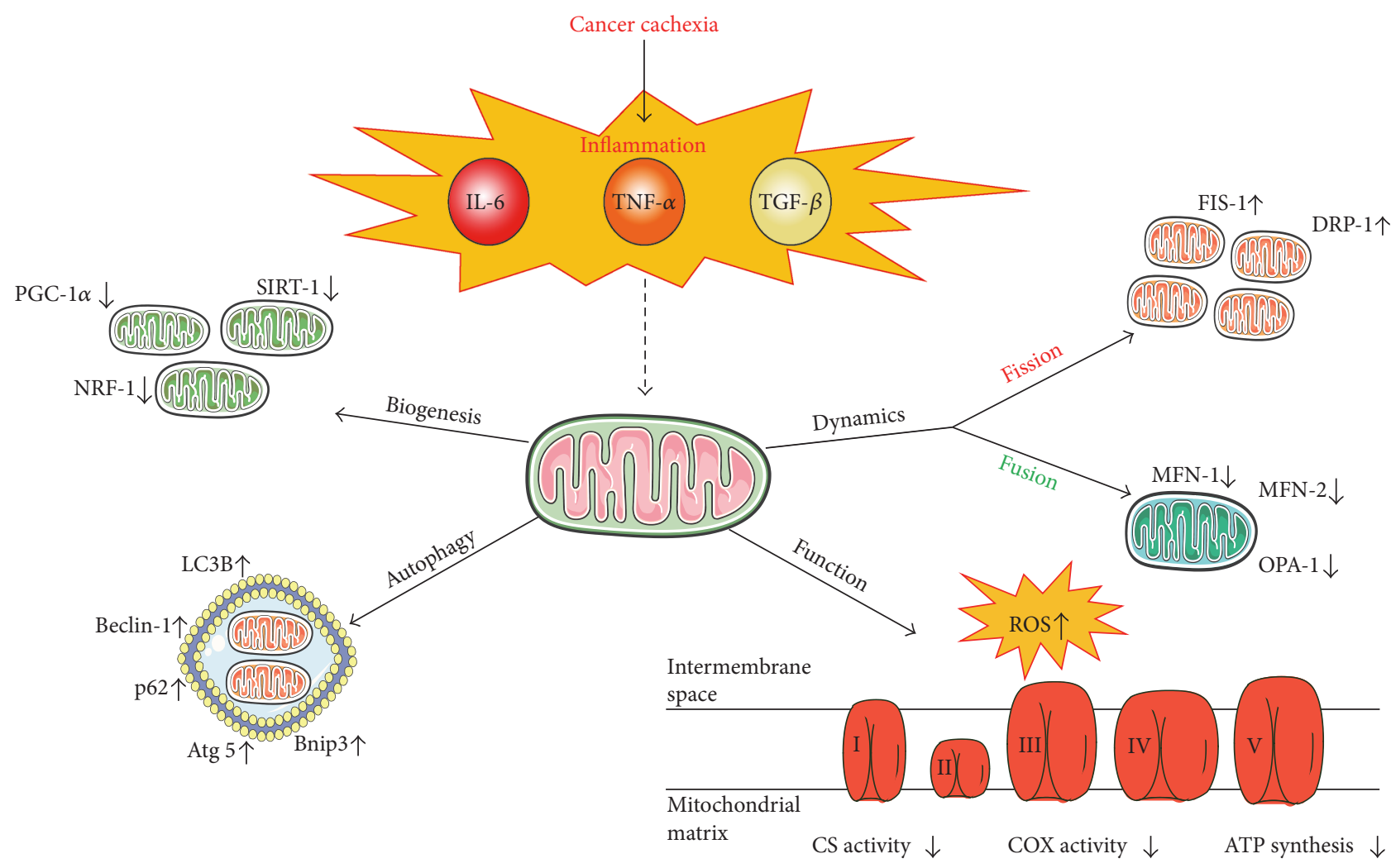

FIGURE 1: Cancer cachexia-induced inflammation regulates mitochondria. Chronic inflammation during cancer cachexia is associated with increased circulatory proinflammatory cytokines IL-6, TNF- $\alpha$, and TGF- $\beta$. Chronic inflammation through these cytokines has been demonstrated to decrease mitochondrial biogenesis through decreased activation of PGC-1a, NRF-1, and Sirt-1. Increased autophagy is apparent in cachectic muscle through inducing LC3B, Beclin-1, p62, Atg 5, and Bnip3 and dysregulating dynamics shown by increased FIS-1 and Drp-1 and decreased MFN-1, MFN-2, and OPA-1. These factors contribute to decreased mitochondrial function and ATP synthesis. The figure was made with Servier Medical Art (http://www.servier.com/Powerpoint-image-bank).

skeletal muscle exhibits decreased cytochrome c oxidase enzymatic activity and oxygen consumption $[70,71]$.

Inflammatory signaling has been linked to cancerinduced mitochondrial dysfunction in skeletal muscle [12]. Specifically, activation of either NF- $\kappa$ B, STAT3, or Smad3 signaling has been associated with cancer-induced muscle mitochondria dysfunction in tumor-bearing mice. In vivo and in vitro analysis of Lewis lung carcinoma-driven cachexia demonstrated decreased muscle ATP synthesis rates and decreased mitochondrial electron flow with associative increases in TNF- $\alpha[68,72]$. Furthermore, inhibiting NF- $\kappa$ B signaling improved diaphragm mitochondrial respiration in mice bearing P07 lung-derived tumors [70]. The IL-6 signaling pathway has also been linked to muscle mitochondrial function with cachexia [12]. STAT3 accumulation in isolated liver and heart mitochondria negatively regulates mitochondrial respiration and ATP production through binding to Complex I in the inner mitochondrial membrane and interacting with retinoid-interferon-induced mortality (GRIM) 19 [73]. Reduced enzyme activity in isolated skeletal muscle mitochondria is demonstrated in mice with elevated Smad 3 signaling [71]. Currently, our understanding of muscle mitochondrial respiration during cancer cachexia is extremely limited due to the dearth of published studies and the heterogeneity of the preclinical cancer cachexia models used in these investigations. However, further mechanistic inquiries into both the drivers of mitochondrial dysfunction and the ramifications of this dysfunction for muscle wasting and functional decline are warranted.

Mitochondrial dysfunction has been tightly associated with excess production of reactive oxygen species (ROS) [72]. While ROS generation is involved in muscle cellular signaling that supports cell homeostasis [72], chronically elevated ROS can initiate DNA damage, protein oxidation, and apoptosis $[70,74,75]$. To this end, substantial evidence points to increased ROS production in cachectic skeletal muscle [76-78]. The role for ROS to promote skeletal muscle dysfunction and atrophy is well established and has been reviewed extensively [79-85]. Although elevated ROS has been identified in wasting skeletal muscle, it has not yet been determined if ROS initiates muscle catabolism in cancer cachexia or is a consequence of the wasting process [82].

3.2. Mitochondrial Dynamics. Understanding skeletal muscle mitochondrial dynamics during cancer cachexia has become an extremely active area of investigation. While initial studies focused on describing changes to mitochondrial dynamics in cachectic muscle, recent research has begun to elucidate the drivers of disrupted mitochondrial dynamics in cachectic muscle and the ramifications this disruption has on muscle 
mass loss and metabolic dysfunction [12, 13, 25]. The interconnected muscle mitochondrial network undergoes tightly regulated processes related to fusion and fission, which are coordinated to influence mitochondrial homeostasis $[13,86,87]$. The fusion of mitochondria induces extension of the mitochondrial network thought to increase energy efficiency and increase ATP production [20]. Conversely, the process of fission involves the fragmentation of mitochondria and segregates damaged areas of the mitochondrial network that may be dysfunctional, allowing for their removal [86-90]. Mitochondrial dysfunction can result from the disrupted coordination of fission and fusion processes; several preclinical models of cancer cachexia and cancer patients have demonstrated altered indices of mitochondrial fission and fusion [91-93].

Mitochondrial dynamics' processes have been extensively studied and characterized both in vivo and in vitro and have been previously reviewed [13, 90, 94, 95]. The fusion process is regulated by mitofusin 1 and 2 (MFN1, MFN-2) and optic atrophy protein 1 (OPA1) [88, 95]. While these proteins are similar in structure, their functions are thought to be nonredundant. MFN-1 regulates GTP tethering whereas MFN-2 regulates the assembly of the fusion complexes $[12,13]$. OPA-1 is expressed as several different isoforms and is necessary for the regulation of fusion GTP tethering in conjunction with MFN-1 [87]. The loss of mitochondrial fusion has detrimental effects in skeletal muscle shown by genetic knockout of MFN-1 and 2 resulting in muscle atrophy and reduced mitochondrial DNA (mtDNA) [96].

Circulating IL- 6 and muscle STAT3 signaling have been linked to suppressed MFN-1 expression in cachectic muscle. Systemic IL-6 overexpression in $A p c^{\mathrm{Min} /+}$ suppressed MFN1 expression, but was rescued by administration of an IL- 6 receptor antibody [92]. Additionally, IL-6 administration to cultured myotubes increased STAT3 activation and suppressed MFN-2 in a dose-dependent manner [92]. Similarly, TNF- $\alpha$ was able to decrease myotube MFN-2 expression associated with elevated ROS and reduced ATP production [97].

Mitochondrial fission is necessary for skeletal muscle mitochondria maintenance and quality [13, 86, 89, 90, 98]. Mitochondrial fission machinery is controlled by the GTPase cytosolic dynamin-related protein 1 (DRP-1) which can translocate to the outer mitochondrial membrane and develop active fission sites [13, 86, 87]. DRP-1 can be regulated by phosphorylation and sumoylation by small ubiquitin-related modifiers (SUMOs) [13]. Fission protein 1 (FIS-1) is proposed to be required for mitochondrial division as it serves to recruit DRP-1 to the outer mitochondrial membrane [87]. Accelerated fission results in proapoptotic signals that lead to mitochondria isolation from the network and reduces its ATP efficiency [90]. Interestingly, accelerated mitochondrial fission is associated with AMPK activation, which can stimulate mitochondrial biogenesis in healthy muscle $[86,99]$. However, while accelerated fission is often regarded as a sign of mitochondrial dysfunction in inflammatory diseases $[12,77,89,92]$, failure to undergo fission will result in mitochondrial dysfunction and muscle atrophy $[86,89,92]$ (Figure 2).

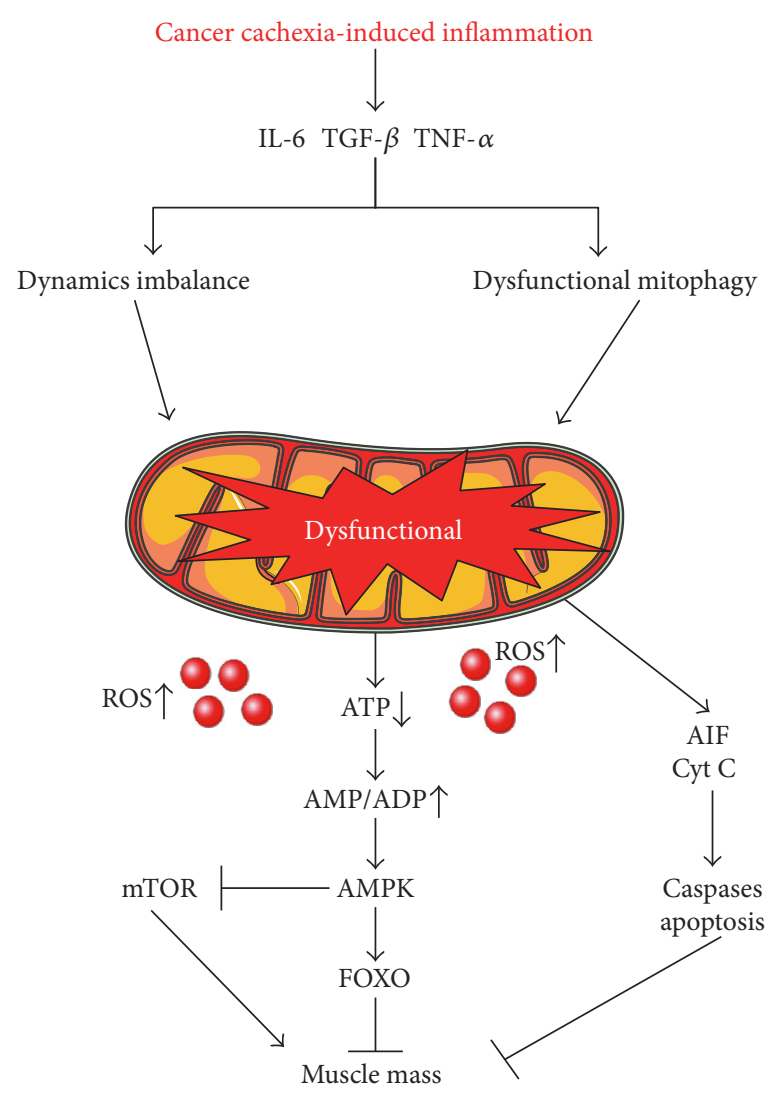

Figure 2: Mitochondrial dysfunction in skeletal muscle negatively regulates muscle mass. Elevated IL- 6 , TNF- $\alpha$, and TGF- $\beta$ during cancer cachexia disrupt mitochondrial homeostasis leading to dysfunction mitochondria. Dysfunctional mitochondria release aberrant amounts of reactive oxygen species (ROS) and decrease ATP production. This leads to chronic activation of AMPK to negatively regulate protein synthesis causing decreased muscle mass. The figure was made with Servier Medical Art (http://www. servier.com/Powerpoint-image-bank).

Although evidence suggests an important role, the direct effects of inflammation on mitochondrial fission continue to be established. Systemic IL-6 overexpression in $\mathrm{Apc}^{\mathrm{Min} /+}$ mice had elevated FIS- 1 protein levels prior to the onset of cachexia [92]. Interestingly, IL-6-induced muscle FIS-1 expression is not selective to muscle phenotype as it occurs equally in both highly oxidative and highly glycolytic fibers $[77,92]$. While a direct link between TNF- $\alpha$ and skeletal muscle mitochondrial fission is not well established, the TNF- $\alpha$ induction of ROS provides intriguing rationale. Interestingly, overexpression of FIS-1 in healthy animals has been demonstrated to be proapoptotic and is tightly associated with accelerated production of ROS [86]. However, it is not well understood if elevated ROS production is causal or consequence of disrupted mitochondrial dynamics in cachectic muscle [100].

3.3. Mitophagy. Hyperactivation of cellular degradation pathways has become an established target of chronic inflammatory conditions [101, 102]. Autophagy in cachectic muscle has become widely investigated for the regulation of skeletal muscle mass loss and disrupted metabolism $[38,103,104]$. 
Autophagy is a highly conserved cellular process that contributes to the lysosomal degradation of proteins and organelles (including mitochondria) that are either dysfunctional, damaged, long lived, or misfolded [88, 105]. The process of autophagy consists of a small portion of the cytoplasm that includes organelles or proteins being sequestered by a phagophore to form an autophagosome. This autophagosome will then fuse with the lysosome to become an autolysosome which then degrades the cellular cargo contained within it. These processes require a family of proteins known as autophagy-related genes (Atgs) which are important in the signaling and regulation of autophagy [106-111]. Autophagy can be both a nonselective process (e.g., starvation) and a highly selective process that degrades specific organelles such as mitochondria which has been termed mitophagy $[12,106$, 107]. The selectivity of this process can be determined by specific proteins, p62 and BCL2 interacting protein 3 (Bnip3), which have cargo-binding domains and LC3-interacting domains which are responsible for recruitment and binding of autophagosome proteins [13, 90, 112]. The autophagic removal of damaged and dysfunctional mitochondria, mitophagy, is critical for maintaining a healthy network of mitochondria. Failure of these processes can lead to an accumulation of damaged mitochondria which can negatively regulate metabolism and mass [13, 110, 113].

Increased lysosomal protease activity, indicative of accelerated autophagy flux, has been reported in cachectic muscle from tumor-bearing mice $[113,114]$. Interestingly, circulating branched chain amino acids are elevated in cancer patients prior to weight loss, suggesting accelerated autophagy is an early event in cachexia development [115]. Tumor growth is associated with a reduced nutrient availability, and it has been suggested that tumor-derived factors can accelerate mitophagy [116]. Accelerated mitophagy has the potential to contribute to skeletal muscle mitochondrial dysfunction $[92,117,118]$. Skeletal muscle from cancer patients and preclinical models of cancer cachexia $\left(A p c^{M i n /+}, C 26\right.$, and LLC) have demonstrated accelerated mitophagy indices [92, 101, 104, 119-121]. Muscle mitophagy can occur through an AMPK, FOXO, and mTORC1 signaling axis, which are established regulators of both muscle metabolism and mass; the cachectic environment also disrupts this signaling axis [110, 121, 122] (Figure 2). Tumor necrosis receptor factor 6 (TRAF6) is a potent inducer of mitophagy, and TRAF6 deletion can prevent cancer-induced muscle mass in tumor-bearing mice [123]. Interestingly, both Activin A and TWEAK have identified roles in the modulation of LC3, potentially indicating disrupted mitophagy [56, 67]. Recently, tumor-derived factors released into circulation were shown to induce mitophagy in skeletal muscle through IL-6-dependent signaling [124]. The autophagy inducing bioactivity of serum collected from gastrointestinal and lung cancer patients was significantly correlated to weight loss, but was normalized with the administration of an IL-6 receptor antibody [124]. Together, current evidence suggests that during cancer cachexia, tumor-secreted IL-6 has an important role in mitophagy regulation. Further, work is warranted to determine if disrupted mitophagy regulation is a viable therapeutic target for cancer-induced muscle wasting or if mitophagy is being induced in cachectic muscle to correct other metabolic dysfunctions.

3.4. Mitochondrial Biogenesis. Muscle adaptation to increased and decreased use provides a clear demonstration that healthy skeletal muscle fiber's mitochondria content is plastic and reflects the fiber's energy requirements [13, 125-127]. However, chronic inflammation can create an environment that disrupts this regulation to incite the loss of muscle oxidative metabolic capacity [128-131]. Mitochondrial biogenesis is a critical process for maintaining the necessary mitochondria content to meet energy demands $[13,125,127,132]$. The peroxisome-proliferator gamma-activated receptor (PGC-1) has been extensively examined as a critical regulator of muscle mitochondrial biogenesis. There are several PGC-1 isoforms, and each has significant but independent roles in oxidative metabolism. PGC- $1 \alpha 4$ regulates muscle protein synthesis through IGF-1 and myostatin signaling cascades $[133,134]$. PGC-1 $\beta$ can regulate myosin heavy-chain isoform expression, and increased expression induces an oxidative muscle phenotype [135]. PGC- $1 \alpha$ can induce nuclear response factors (NRF-1, NRF-2) and mitochondrial transcription factor A (Tfam) transcription, which regulate mitochondrial biogenesis $[125-127,136]$. Moreover, PGC- $1 \alpha$ loss results in reduced muscle mitochondrial content and ATP production [137-139].

Increased PGC- $1 \alpha$ expression is protective against muscle atrophy in aging, decreased use, and inflammatory cytokine administration. However, the limited number of investigations in preclinical cancer cachexia models is equivocal [140-143]. While suppressed PGC- $1 \alpha$ expression is consistently reported in cachectic skeletal muscle [77, 92, 144], overexpression was not sufficient to prevent Lewis lung carcinoma- (LLC-) induced muscle wasting [145]. Interestingly, PGC- $1 \alpha$ overexpression could stimulate mitochondrial biogenesis in the cachectic muscle, indicating that the pathways to induce mitochondrial biogenesis were functional in the cachectic environment. Regulators of PGC- $1 \alpha$ activity are also major determinants of muscle metabolic capacity in both healthy and cachectic muscle [146]. AMPK, an energy stress sensor, regulates muscle oxidative metabolism through PGC$1 \alpha$-regulated biogenesis and ULK-dependent mitophagy [147-149]. In healthy muscle, activated AMPK can stimulate mitochondrial biogenesis and has been demonstrated as therapeutic in type II diabetes $[149,150]$. Muscle AMPK is chronically activated in some preclinical cancer cachexia models, however, fails to induce mitochondrial biogenesis. Interestingly, chronic AMPK activation in cachectic muscle may have a role in the suppression of muscle protein synthesis [151]. Circulating IL-6 has been associated with muscle AMPK activation in the cachectic $A p c^{\mathrm{Min} /+}$ mouse. IL- 6 overexpression in tumor-bearing mice can activate AMPK and reduce PGC- $1 \alpha$ expression, whereas IL- 6 receptor antibody administration attenuates cancer-induced AMPK activation [151]. Although in vivo evidence for the direct effects of IL6 signaling on cachectic muscle AMPK activation is lacking, IL-6 administration to skeletal muscle myotubes can directly activate AMPK [151]. Further research is needed to understand the disrupted feedback caused by the cachectic 


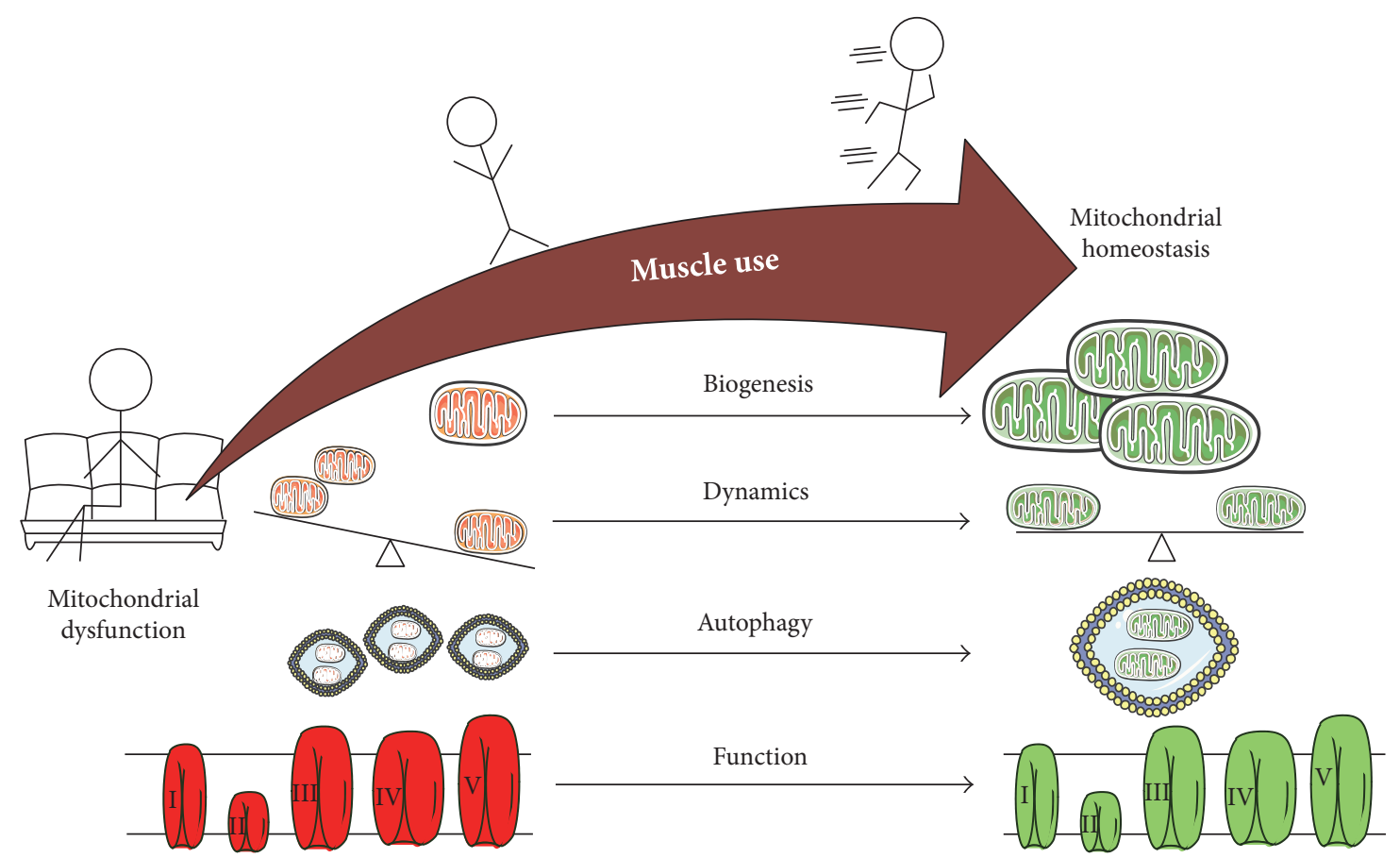

FIGURE 3: Increased muscle use improves skeletal muscle mitochondrial homeostasis in healthy and cachectic conditions. Sedentary behavior or muscle disuse is associated with decreased mitochondrial biogenesis, improper balance of mitochondrial dynamics, dysregulation of autophagy, and mitochondrial dysfunction. Increasing muscle use by reducing sedentary behavior or exercise will increase mitochondrial biogenesis, regulate mitochondrial dynamics, improve autophagic flux, and improve mitochondrial function and ATP efficiency. Overall increased muscle use will lead to skeletal muscle mitochondrial and metabolic homeostasis. The figure was made with Servier Medical Art (http://www.servier.com/Powerpoint-image-bank).

environment that uncouples AMPK signaling from mitochondrial biogenesis.

Activin A and myostatin have the potential to also disrupt mitochondrial biogenesis. Ge et al. demonstrated that the lack of Smad3 signaling resulted in decreased NRF and Tfam activation [152]. However, Smad3 activation via the TGF- $\beta$ super family in cachectic skeletal muscle remains to be determined. Interestingly, both Activin A and TWEAK were able to disrupt mitochondrial biogenesis by reducing PGC1- $\alpha[67,153]$. Further research is needed to establish if this mitochondrial biogenesis suppression is a therapeutic target for either preventing muscle mass loss or improving metabolic health during cancer cachexia.

\section{Exercise Countermeasures to Cancer-Induced Mitochondrial Dysfunction}

The capacity to regenerate from injury and adapt to altered use are defining features of skeletal muscle that also provide optimism for therapeutic interventions for cachectic muscle. Exercise has shown to be beneficial in diabetes, COPD, and $\mathrm{CHF}$ and continues to show beneficial results in cancer patients as well [154, 155]. Activity level can dramatically impact skeletal muscle mass and metabolism [88, 156]. Increased muscle activity can also induce a more oxidative muscle phenotype by increasing mitochondria content and function [95, 157-159]. Increased muscle use can positively impact muscle mass, and the extent of this change is dependent on the exercise type, intensity, duration, and frequency $[5,160]$. The metabolic plasticity of muscle is reinforced by the dramatic alterations that occur to skeletal muscle after an acute bout of exercise [88, 156, 157]. Increasing the muscle metabolic demand with exercise can stimulate mitochondrial biogenesis to increase mitochondrial content and function $[138,139,161]$. Cachectic muscle from tumor-bearing mice subjected to an acute bout of low frequency electrical stimulation maintains the capacity to activate genes responsible for mitochondrial biogenesis, PGC$1 \alpha$, NRF-1, and Tfam [144]. However, cachectic muscle had deficits in the acute activation of protein expression after a single bout of stimulated concentric contractions, which could be rescued by systemic inhibition of inflammatory signaling [144].

Decreased muscle use, either by unloading or extreme sedentary behavior, can induce a shift to a more glycolytic phenotype, coinciding with decreased mitochondrial content and function and muscle atrophy [155]. Cancer patients commonly suffer from excessive fatigue prior to and during treatments $[5,6,162]$. This fatigue is accompanied by a dramatic decrease in physical activity and the ability to perform activities of daily living (ADLs) [5, 163-165]. Preclinical cancer models also have shown that cachectic mice undergo limited volitional activity [166, 167]. However, minimizing sedentary time and using alternative muscle contraction methods may serve as a first line of action to attenuate cachexia-induced decrements in muscle mitochondria 
[168-171] (Figure 3). IL-6 overexpression in tumor-bearing mice was not able to induce muscle mass loss and metabolic changes when they were regularly exercised on a treadmill [172]. It is interesting to speculate if disuse alters the muscle sensitivity to the cachectic environment, causing a more rapid decline in muscle metabolic function and mass. Conversely, research is needed to determine if muscle contraction or exercise serves to desensitize the muscle to the cachectic environment.

Exercise also regulates mitochondrial dynamics, increasing both fission and fusion. This is thought to aid in mitochondrial turnover and improve efficiency (Figure 3). Similarly, autophagy flux increases after an acute bout of exercise. IL-6 overexpression in exercising tumor-bearing mice was resistant to muscle mass loss and metabolic changes [172]. Cachectic muscle in tumor-bearing mice also retains the capacity to respond to repeated bouts of stimulated eccentric contractions. Cachectic muscle in $A p c^{\mathrm{Min} /+}$ mice undergoing 7 bouts of eccentric contractions increased muscle succinate dehydrogenase activity and decreased AMPK signaling [173]. Exercise training is implicated as a potential therapeutic to either prevent or reverse muscle wasting. It is evident from preclinical studies that cachectic muscle maintains the ability to robustly respond to an acute bout of exercise or contraction. Further work is needed to determine if repeated bouts of exercise can confer the metabolic health benefits of exercise after the development of cancer cachexia [139, 174].

Physical activity and contraction has been established as a potent regulator of mitophagy and may possess the potential to correct or attenuate dysfunction mitophagy processes in cachectic muscle $[88,105,175-179]$. In C26 tumorimplanted mice, voluntary wheel running attenuated cachexia-induced p62 and LC3 II/I accumulation indicating improved mitophagy [179]. Additionally, AMPK activation via AICAR suppressed 662 accumulation through promotion of mitophagy and accelerating the turnover of p62 accumulation in cachectic muscle [179]. While there is growing evidence for mitophagic processes in the regulation of cancer cachexia, additional studies are warranted to establish a direct role for inflammation in the regulation of these processes and to clearly examine mitophagy flux in vivo. Additionally, the role of exercise and/or muscle contraction in the regulation of mitophagy in diseased or chronically inflamed muscle may prove to be a powerful therapeutic for the restoration of mitophagic balance in cachectic muscle. Clearly, further research is warranted to examine the complex interaction between cancer-induced inflammation, muscle contraction, and muscle disuse for maintenance or improvement of cachectic muscle oxidative metabolism.

\section{Conclusions}

Disrupted mitochondrial homeostasis contributes to the loss of functional capacity in cancer patients and can negatively impact the quality of life and survival. Skeletal muscle mitochondria not only regulate oxidative metabolism but also proteostasis and muscle mass maintenance. The role of mitochondria in skeletal muscle wasting during cancer cachexia has emerged as novel investigative target in cachexia studies. There is a clear relationship between inflammation and mitochondrial dysfunction related to dynamics, mitophagy, and biogenesis. While the alterations to mitochondrial dynamics during cachexia appear evident, a more mechanistic approach is necessary to understand regulatory mechanisms and functional outcomes. A growing body of research suggests an important therapeutic strategy involving the reduction of muscle disuse and increasing muscle contractile activity for the maintenance of skeletal muscle metabolic health, even in the presence of a cachectic environment. To this end, analysis of functional and metabolic outcomes, muscle strength, and fatigability are necessary to understand the totality of the cachectic condition.

\section{Conflicts of Interest}

The authors declare that there is no conflict of interest regarding the publication of this paper.

\section{Acknowledgments}

This work was supported by the National Institutes of Health, grant no. R01-CA121249 from the National Cancer Institute (JAC) and grant no. P20 RR-017698 from the National Center for Research (JAC).

\section{References}

[1] J. M. Argiles, S. Busquets, M. Toledo, and F. J. López-Soriano, "The role of cytokines in cancer cachexia," Current Opinion in Supportive and Palliative Care, vol. 3, no. 4, pp. 263-268, 2009.

[2] K. C. Fearon, "Cancer cachexia: developing multimodal therapy for a multidimensional problem," European Journal of Cancer, vol. 44, no. 8, pp. 1124-1132, 2008.

[3] W. J. Evans, J. E. Morley, J. Argilés et al., "Cachexia: a new definition," Clinical Nutrition, vol. 27, no. 6, pp. 793-799, 2008.

[4] M. J. Tisdale, "Cachexia in cancer patients," Nature Reviews. Cancer, vol. 2, no. 11, pp. 862-871, 2002.

[5] S. al-Majid and D. O. McCarthy, "Cancer-induced fatigue and skeletal muscle wasting: the role of exercise," Biological Research for Nursing, vol. 2, no. 3, pp. 186-197, 2001.

[6] P. Stone, J. Hardy, K. Broadley, A. J. Tookman, A. Kurowska, and R. A'Hern, "Fatigue in advanced cancer: a prospective controlled cross-sectional study," British Journal of Cancer, vol. 79, no. 9-10, pp. 1479-1486, 1999.

[7] P. O'Gorman, D. C. McMillan, and C. S. McArdle, "Longitudinal study of weight, appetite, performance status, and inflammation in advanced gastrointestinal cancer," Nutrition and Cancer, vol. 35, no. 2, pp. 127-129, 1999.

[8] S. Haehlingvon and S. D. Anker, "Prevalence, incidence and clinical impact of cachexia: facts and numbers-update 2014," Journal of Cachexia, Sarcopenia and Muscle, vol. 5, no. 4, pp. 261-263, 2014.

[9] J. F. Christensen, L. W. Jones, J. L. Andersen, G. Daugaard, M. Rorth, and P. Hojman, "Muscle dysfunction in cancer patients," Annals of Oncology, vol. 25, no. 5, pp. 947-958, 2014. 
[10] M. L. Winningham, L. M. Nail, M. B. Burke et al., "Fatigue and the cancer experience: the state of the knowledge," Oncology Nursing Forum, vol. 21, no. 1, pp. 23-36, 1994.

[11] R. R. Wolfe, "The underappreciated role of muscle in health and disease," The American Journal of Clinical Nutrition, vol. 84, no. 3, pp. 475-482, 2006.

[12] J. A. Carson, J. P. Hardee, and B. N. VanderVeen, "The emerging role of skeletal muscle oxidative metabolism as a biological target and cellular regulator of cancer-induced muscle wasting," Seminars in Cell \& Developmental Biology, vol. 54, pp. 53-67, 2016.

[13] V. Romanello and M. Sandri, "Mitochondrial quality control and muscle mass maintenance," Frontiers in Physiology, vol. 6, p. 422, 2015.

[14] C. M. Kummitha, S. C. Kalhan, G. M. Saidel, and N. Lai, "Relating tissue/organ energy expenditure to metabolic fluxes in mouse and human: experimental data integrated with mathematical modeling," Physiological Reports, vol. 2, no. 9, 2014.

[15] J. M. Argiles, N. Campos, J. M. Lopez-Pedrosa, R. Rueda, and L. Rodriguez-Mañas, "Skeletal muscle regulates metabolism via interorgan crosstalk: roles in health and disease," Journal of the American Medical Directors Association, vol. 17, no. 9, pp. 789-796, 2016.

[16] D. F. Wilson, "Energy metabolism in muscle approaching maximal rates of oxygen utilization," Medicine and Science in Sports and Exercise, vol. 27, no. 1, pp. 54-59, 1995.

[17] R. I. Close, "Dynamic properties of mammalian skeletal muscles," Physiological Reviews, vol. 52, no. 1, pp. 129-197, 1972.

[18] P. Deng and C. M. Haynes, "Mitochondrial dysfunction in cancer: potential roles of ATF5 and the mitochondrial UPR," Seminars in Cancer Biology, 2017.

[19] K. Prasai, "Regulation of mitochondrial structure and function by protein import: a current review," Pathophysiology, 2017.

[20] M. Bordi, F. Nazio, and S. Campello, "The close interconnection between mitochondrial dynamics and mitophagy in cancer," Frontiers in Oncology, vol. 7, p. 81, 2017.

[21] M. L. Boland, A. H. Chourasia, and K. F. Macleod, "Mitochondrial dysfunction in cancer," Frontiers in Oncology, vol. 3, p. 292, 2013.

[22] A. Picca, A. M. S. Lezza, C. Leeuwenburgh et al., "Fueling inflamm-aging through mitochondrial dysfunction: mechanisms and molecular targets," International Journal of Molecular Sciences, vol. 18, no. 5, 2017.

[23] R. Thibault, S. Chanséaume, K. Azarnoush et al., "Mitochondrial protein synthesis is increased in oxidative skeletal muscles of rats with cardiac cachexia," Nutrition Research, vol. 34, no. 3, pp. 250-257, 2014.

[24] C. M. Julienne, J. F. Dumas, C. Goupille et al., "Cancer cachexia is associated with a decrease in skeletal muscle mitochondrial oxidative capacities without alteration of ATP production efficiency," Journal of Cachexia, Sarcopenia and Muscle, vol. 3, pp. 265-275, 2012.

[25] J. M. Argiles, F. J. Lopez-Soriano, and S. Busquets, "Muscle wasting in cancer: the role of mitochondria," Current Opinion in Clinical Nutrition and Metabolic Care, vol. 18, no. 3, pp. 221-225, 2015.

[26] R. Vitorino, D. Moreira-Goncalves, and R. Ferreira, "Mitochondrial plasticity in cancer-related muscle wasting: potential approaches for its management," Current Opinion in Clinical Nutrition and Metabolic Care, vol. 18, no. 3, pp. 226-233, 2015.

[27] V. Adams, S. D. Anker, and G. Schuler, "Muscle metabolism and exercise capacity in cachexia," Current Pharmaceutical Design, vol. 17, no. 35, pp. 3838-3845, 2011.

[28] B. M. Roberts, G. S. Frye, B. Ahn, L. F. Ferreira, and A. R. Judge, "Cancer cachexia decreases specific force and accelerates fatigue in limb muscle," Biochemical and Biophysical Research Communications, vol. 435, no. 3, pp. 488-492, 2013.

[29] J. A. Carson and K. A. Baltgalvis, "Interleukin 6 as a key regulator of muscle mass during cachexia," Exercise and Sport Sciences Reviews, vol. 38, no. 4, pp. 168-176, 2010.

[30] J. L. Chen, T. D. Colgan, K. L. Walton, P. Gregorevic, and C. A. Harrison, "The TGF-beta signalling network in muscle development, adaptation and disease," Advances in Experimental Medicine and Biology, vol. 900, pp. 97-131, 2016

[31] A. Loumaye, M. Barsyde, M. Nachit et al., "Role of Activin A and myostatin in human cancer cachexia," The Journal of Clinical Endocrinology and Metabolism, vol. 100, no. 5, pp. 2030-2038, 2015.

[32] T. C. Mueller, J. Bachmann, O. Prokopchuk, H. Friess, and M. E. Martignoni, "Molecular pathways leading to loss of skeletal muscle mass in cancer cachexia-can findings from animal models be translated to humans?," BMC Cancer, vol. 16, p. 75, 2016.

[33] J. K. Onesti and D. C. Guttridge, "Inflammation based regulation of cancer cachexia," BioMed Research International, vol. 2014, Article ID 168407, 7 pages, 2014.

[34] H. J. Patel and B. M. Patel, "TNF-alpha and cancer cachexia: molecular insights and clinical implications," Life Sciences, vol. 170, pp. 56-63, 2017.

[35] M. Pal, M. A. Febbraio, and M. Whitham, "From cytokine to myokine: the emerging role of interleukin-6 in metabolic regulation," Immunology and Cell Biology, vol. 92, no. 4, pp. 331-339, 2014.

[36] M. B. Reid and Y. P. Li, "Tumor necrosis factor-alpha and muscle wasting: a cellular perspective," Respiratory Research, vol. 2, no. 5, pp. 269-272, 2001.

[37] J. Rodriguez, B. Vernus, I. Chelh et al., "Myostatin and the skeletal muscle atrophy and hypertrophy signaling pathways," Cellular and Molecular Life Sciences, vol. 71, no. 22, pp. 4361-4371, 2014

[38] M. Sandri, "Signaling in muscle atrophy and hypertrophy," Physiology (Bethesda), vol. 23, pp. 160-170, 2008.

[39] J. E. Belizario, C. C. Fontes-Oliveira, J. P. Borges, J. A. Kashiabara, and E. Vannier, "Skeletal muscle wasting and renewal: a pivotal role of myokine IL-6," Spring, vol. 5, p. 619, 2016.

[40] T. R. Flint, T. Janowitz, C. M. Connell et al., "Tumorinduced IL-6 reprograms host metabolism to suppress anti-tumor immunity," Cell Metabolism, vol. 24, no. 5, pp. 672-684, 2016.

[41] A. Bonetto, J. K. Kays, V. A. Parker et al., "Differential bone loss in mouse models of colon cancer cachexia," Frontiers in Physiology, vol. 7, p. 679, 2016.

[42] A. A. Narsale and J. A. Carson, "Role of interleukin-6 in cachexia: therapeutic implications," Current Opinion in Supportive and Palliative Care, vol. 8, no. 4, pp. 321-327, 2014. 
[43] B. K. Pedersen, "Muscular interleukin-6 and its role as an energy sensor," Medicine and Science in Sports and Exercise, vol. 44, no. 3, pp. 392-396, 2012.

[44] N. Fortunati, R. Manti, N. Birocco et al., "Pro-inflammatory cytokines and oxidative stress/antioxidant parameters characterize the bio-humoral profile of early cachexia in lung cancer patients," Oncology Reports, vol. 18, no. 6, pp. 1521-1527, 2007.

[45] K. A. Baltgalvis, F. G. Berger, M. M. Peña, J. M. Davis, J. P. White, and J. A. Carson, "Muscle wasting and interleukin-6induced atrogin-I expression in the cachectic $A p c^{M i n /+}$ mouse," Pflügers Archiv, vol. 457, no. 5, pp. 989-1001, 2009.

[46] D. N. Seto, S. C. Kandarian, and R. W. Jackman, "A key role for leukemia inhibitory factor in C26 cancer cachexia," The Journal of Biological Chemistry, vol. 290, no. 32, pp. 1997619986, 2015.

[47] M. J. Puppa, S. Gao, A. A. Narsale, and J. A. Carson, "Skeletal muscle glycoprotein 130 's role in Lewis lung carcinomainduced cachexia," The FASEB Journal, vol. 28, no. 2, pp. 998-1009, 2014.

[48] L. Cron, T. Allen, and M. A. Febbraio, "The role of gp130 receptor cytokines in the regulation of metabolic homeostasis," The Journal of Experimental Biology, vol. 219, Part 2, pp. 259-265, 2016.

[49] A. Bonetto, T. Aydogdu, X. Jin et al., "JAK/STAT3 pathway inhibition blocks skeletal muscle wasting downstream of IL6 and in experimental cancer cachexia," American Journal of Physiology. Endocrinology and Metabolism, vol. 303, no. 3, pp. E410-E421, 2012.

[50] A. Bonetto, T. Aydogdu, N. Kunzevitzky et al., "STAT3 activation in skeletal muscle links muscle wasting and the acute phase response in cancer cachexia," PloS One, vol. 6, no. 7, article e22538, 2011.

[51] A. Miller, L. McLeod, S. Alhayyani et al., "Blockade of the IL-6 trans-signalling/STAT3 axis suppresses cachexia in Kras-induced lung adenocarcinoma," Oncogene, vol. 36, no. 21, pp. 3059-3066, 2017.

[52] J. P. White, J. W. Baynes, S. L. Welle et al., "The regulation of skeletal muscle protein turnover during the progression of cancer cachexia in the Apc $c^{\text {min/+ }}$ mouse," PLoS One, vol. 6, no. 9 , article e24650, 2011.

[53] A. Bonetto, T. Aydogdu, X. Jin et al., "JAK/STAT3 pathway inhibition blocks skeletal muscle wasting downstream of IL6 and in experimental cancer cachexia," American Journal of Physiology Endocrinology and Metabolism, vol. 303, no. 3, pp. E410-E421, 2012.

[54] M. B. Reid, J. Lannergren, and H. Westerblad, "Respiratory and limb muscle weakness induced by tumor necrosis factor-alpha: involvement of muscle myofilaments," American Journal of Respiratory and Critical Care Medicine, vol. 166, no. 4, pp. 479-484, 2002.

[55] A. H. Remels, H. R. Gosker, P. Schrauwen et al., "TNF-alpha impairs regulation of muscle oxidative phenotype: implications for cachexia?," The FASEB Journal, vol. 24, no. 12, pp. 5052-5062, 2010.

[56] A. Kumar, S. Bhatnagar, and P. K. Paul, "TWEAK and TRAF6 regulate skeletal muscle atrophy," Current Opinion in Clinical Nutrition and Metabolic Care, vol. 15, no. 3, pp. 233-239, 2012.

[57] M. J. Tisdale, "The ubiquitin-proteasome pathway as a therapeutic target for muscle wasting," The Journal of Supportive Oncology, vol. 3, no. 3, pp. 209-217, 2005.
[58] J. S. Patton, P. M. Peters, J. McCabe et al., "Development of partial tolerance to the gastrointestinal effects of high doses of recombinant tumor necrosis factor-alpha in rodents," The Journal of Clinical Investigation, vol. 80, no. 6, pp. 1587-1596, 1987.

[59] M. C. Stovroff, D. L. Fraker, J. A. Swedenborg, and J. A. Norton, "Cachectin/tumor necrosis factor: a possible mediator of cancer anorexia in the rat," Cancer Research, vol. 48, no. 16, pp. 4567-4572, 1988.

[60] I. L. Bernstein, E. M. Taylor, and K. L. Bentson, "TNFinduced anorexia and learned food aversions are attenuated by area postrema lesions," The American Journal of Physiology, vol. 260, 5 Part 2, pp. R906-R910, 1991.

[61] H. D. Mulligan, S. M. Mahony, J. A. Ross, and M. J. Tisdale, "Weight loss in a murine cachexia model is not associated with the cytokines tumour necrosis factor-alpha or interleukin-6," Cancer Letters, vol. 65, no. 3, pp. 239-243, 1992.

[62] T. Yoshida, A. M. Tabony, S. Galvez et al., "Molecular mechanisms and signaling pathways of angiotensin II-induced muscle wasting: potential therapeutic targets for cardiac cachexia," The International Journal of Biochemistry \& Cell Biology, vol. 45, no. 10, pp. 2322-2332, 2013.

[63] M. E. Benny Klimek, T. Aydogdu, M. J. Link, M. Pons, L. G. Koniaris, and T. A. Zimmers, "Acute inhibition of myostatinfamily proteins preserves skeletal muscle in mouse models of cancer cachexia," Biochemical and Biophysical Research Communications, vol. 391, no. 3, pp. 1548-1554, 2010.

[64] K. T. Murphy, A. Chee, B. G. Gleeson et al., "Antibodydirected myostatin inhibition enhances muscle mass and function in tumor-bearing mice," American Journal of Physiology. Regulatory, Integrative and Comparative Physiology, vol. 301, no. 3, pp. R716-R726, 2011.

[65] T. A. Zimmers, M. V. Davies, L. G. Koniaris et al., "Induction of cachexia in mice by systemically administered myostatin," Science, vol. 296, no. 5572, pp. 1486-1488, 2002.

[66] A. I. Padrao, D. Moreira-Gonçalves, P. A. Oliveira et al., "Endurance training prevents TWEAK but not myostatinmediated cardiac remodelling in cancer cachexia," Archives of Biochemistry and Biophysics, vol. 567, pp. 13-21, 2015.

[67] J. L. Chen, K. L. Walton, H. Qian et al., "Differential effects of IL6 and Activin A in the development of cancer-associated cachexia," Cancer Research, vol. 76, no. 18, pp. 5372-5382, 2016.

[68] C. Constantinou, C. C. Fontes de Oliveira, D. Mintzopoulos et al., "Nuclear magnetic resonance in conjunction with functional genomics suggests mitochondrial dysfunction in a murine model of cancer cachexia," International Journal of Molecular Medicine, vol. 27, no. 1, pp. 15-24, 2011.

[69] D. Antunes, A. I. Padrão, E. Maciel et al., "Molecular insights into mitochondrial dysfunction in cancer-related muscle wasting," Biochimica et Biophysica Acta-Molecular and Cell Biology of Lipids, vol. 1841, no. 6, pp. 896-905, 2014.

[70] C. Fermoselle, E. García-Arumí, E. Puig-Vilanova et al., "Mitochondrial dysfunction and therapeutic approaches in respiratory and limb muscles of cancer cachectic mice," Experimental Physiology, vol. 98, no. 9, pp. 1349-1365, 2013.

[71] A. I. Padrao, P. Oliveira, R. Vitorino et al., "Bladder cancerinduced skeletal muscle wasting: disclosing the role of mitochondria plasticity," The International Journal of Biochemistry \& Cell Biology, vol. 45, no. 7, pp. 1399-1409, 2013. 
[72] J. B. McLean, J. S. Moylan, and F. H. Andrade, "Mitochondria dysfunction in lung cancer-induced muscle wasting in $\mathrm{C} 2 \mathrm{C} 12$ myotubes," Frontiers in Physiology, vol. 5, p. 503, 2014.

[73] J. Wegrzyn, R. Potla, Y. J. Chwae et al., "Function of mitochondrial Stat3 in cellular respiration," Science, vol. 323, no. 5915, pp. 793-797, 2009.

[74] C. C. Fontes-Oliveira, S. Busquets, M. Toledo et al., "Mitochondrial and sarcoplasmic reticulum abnormalities in cancer cachexia: altered energetic efficiency?", Biochimica et Biophysica Acta, vol. 1830, no. 3, pp. 2770-2778, 2013.

[75] A. A. Tzika, C. C. Fontes-Oliveira, A. A. Shestov et al., "Skeletal muscle mitochondrial uncoupling in a murine cancer cachexia model," International Journal of Oncology, vol. 43, no. 3, pp. 886-894, 2013.

[76] Z. Yu, P. Li, M. Zhang, M. Hannink, J. S. Stamler, and Z. Yan, "Fiber type-specific nitric oxide protects oxidative myofibers against cachectic stimuli," PloS One, vol. 3, no. 5, article e2086, 2008.

[77] J. P. White, K. A. Baltgalvis, M. J. Puppa, S. Sato, J. W. Baynes, and J. A. Carson, "Muscle oxidative capacity during IL-6dependent cancer cachexia," American Journal of Physiology. Regulatory, Integrative and Comparative Physiology, vol. 300, no. 2, pp. R201-R211, 2011.

[78] E. Barreiro, B. Puentede la, S. Busquets, F. J. López-Soriano, J. Gea, and J. M. Argilés, "Both oxidative and nitrosative stress are associated with muscle wasting in tumour-bearing rats," FEBS Letters, vol. 579, no. 7, pp. 1646-1652, 2005.

[79] Y. P. Li, Y. Chen, A. S. Li, and M. B. Reid, "Hydrogen peroxide stimulates ubiquitin-conjugating activity and expression of genes for specific E2 and E3 proteins in skeletal muscle myotubes," American Journal of Physiology. Cell Physiology, vol. 285, no. 4, pp. C806-C812, 2003.

[80] F. L. Muller, W. Song, Y. C. Jang et al., "Denervation-induced skeletal muscle atrophy is associated with increased mitochondrial ROS production," American Journal of Physiology. Regulatory, Integrative and Comparative Physiology, vol. 293, no. 3, pp. R1159-R1168, 2007.

[81] D. A. Hood, G. Uguccioni, A. Vainshtein, and D. D'souza, "Mechanisms of exercise-induced mitochondrial biogenesis in skeletal muscle: implications for health and disease," Comprehensive Physiology, vol. 1, no. 3, pp. 1119-1134, 2011.

[82] S. K. Powers, A. J. Smuder, and A. R. Judge, "Oxidative stress and disuse muscle atrophy: cause or consequence?," Current Opinion in Clinical Nutrition and Metabolic Care, vol. 15, no. 3, pp. 240-245, 2012.

[83] S. K. Powers, E. E. Talbert, and P. J. Adhihetty, "Reactive oxygen and nitrogen species as intracellular signals in skeletal muscle," The Journal of Physiology, vol. 589, Part 9, pp. 2129-2138, 2011.

[84] S. K. Powers, M. P. Wiggs, J. A. Duarte, A. M. Zergeroglu, and H. A. Demirel, "Mitochondrial signaling contributes to disuse muscle atrophy," American Journal of Physiology Endocrinology and Metabolism, vol. 303, no. 1, pp. E31E39, 2012.

[85] L. F. Ferreira and M. B. Reid, "Muscle-derived ROS and thiol regulation in muscle fatigue," Journal of Applied Physiology, vol. 104, no. 3, pp. 853-860, 2008.

[86] V. Romanello, E. Guadagnin, L. Gomes et al., "Mitochondrial fission and remodelling contributes to muscle atrophy," The EMBO Journal, vol. 29, no. 10, pp. 1774-1785, 2010.
[87] S. Iqbal and D. A. Hood, "The role of mitochondrial fusion and fission in skeletal muscle function and dysfunction," Frontiers in Bioscience (Landmark Edition), vol. 20, pp. 157-172, 2015.

[88] Z. Yan, V. A. Lira, and N. P. Greene, "Exercise traininginduced regulation of mitochondrial quality," Exercise and Sport Sciences Reviews, vol. 40, no. 3, pp. 159-164, 2012.

[89] S. Wu, F. Zhou, Z. Zhang, and D. Xing, "Mitochondrial oxidative stress causes mitochondrial fragmentation via differential modulation of mitochondrial fission-fusion proteins," The FEBS Journal, vol. 278, no. 6, pp. 941-954, 2011.

[90] R. J. Youle and A. M. v. d. Bliek, "Mitochondrial fission, fusion, and stress," Science, vol. 337, no. 6098, pp. 1062 1065, 2012.

[91] E. Marzetti, M. Lorenzi, F. Landi et al., "Altered mitochondrial quality control signaling in muscle of old gastric cancer patients with cachexia," Experimental Gerontology, vol. 87, Part A, pp. 92-99, 2017.

[92] J. P. White, M. J. Puppa, S. Sato et al., "IL-6 regulation on skeletal muscle mitochondrial remodeling during cancer cachexia in the Apc $c^{\mathrm{Min} /+}$ mouse," Skeletal Muscle, vol. 2, p. 14, 2012.

[93] C. Guido, D. Whitaker-Menezes, Z. Lin et al., "Mitochondrial fission induces glycolytic reprogramming in cancer-associated myofibroblasts, driving stromal lactate production, and early tumor growth," Oncotarget, vol. 3, no. 8, pp. 798-810, 2012.

[94] S. Iqbal, O. Ostojic, K. Singh, A. M. Joseph, and D. A. Hood, "Expression of mitochondrial fission and fusion regulatory proteins in skeletal muscle during chronic use and disuse," Muscle \& Nerve, vol. 48, no. 6, pp. 963-970, 2013.

[95] J. C. Drake, R. J. Wilson, and Z. Yan, "Molecular mechanisms for mitochondrial adaptation to exercise training in skeletal muscle," The FASEB Journal, vol. 30, no. 1, pp. 13-22, 2016.

[96] J. Y. Lee, M. Kapur, M. Li et al., "MFN1 deacetylation activates adaptive mitochondrial fusion and protects metabolically challenged mitochondria," Journal of Cell Science, vol. 127, Part 22, pp. 4954-4963, 2014.

[97] Y. Zhang, L. Yang, Y. F. Gao et al., "MicroRNA-106b induces mitochondrial dysfunction and insulin resistance in $\mathrm{C} 2 \mathrm{C} 12$ myotubes by targeting mitofusin-2," Molecular and Cellular Endocrinology, vol. 381, no. 1-2, pp. 230240, 2013.

[98] H. F. Jheng, P. J. Tsai, S. M. Guo et al., "Mitochondrial fission contributes to mitochondrial dysfunction and insulin resistance in skeletal muscle," Molecular and Cellular Biology, vol. 32, no. 2, pp. 309-319, 2012.

[99] K. Marcinko and G. R. Steinberg, "The role of AMPK in controlling metabolism and mitochondrial biogenesis during exercise," Experimental Physiology, vol. 99, no. 12, pp. 1581-1585, 2014.

[100] T. Hennet, C. Richter, and E. Peterhans, "Tumour necrosis factor-alpha induces superoxide anion generation in mitochondria of L929 cells," The Biochemical Journal, vol. 289, Part 2, pp. 587-592, 1993.

[101] J. M. McClung, A. R. Judge, S. K. Powers, and Z. Yan, “p38 MAPK links oxidative stress to autophagy-related gene expression in cachectic muscle wasting," American Journal of Physiology. Cell Physiology, vol. 298, no. 3, pp. C542C549, 2010. 
[102] E. Puig-Vilanova, D. A. Rodriguez, J. Lloreta et al., "Oxidative stress, redox signaling pathways, and autophagy in cachectic muscles of male patients with advanced COPD and lung cancer," Free Radical Biology and Medicine, vol. 79, pp. 91-108, 2015.

[103] Y. Luo, J. Yoneda, H. Ohmori et al., "Cancer usurps skeletal muscle as an energy repository," Cancer Research, vol. 74, no. 1, pp. 330-340, 2014.

[104] E. E. Talbert, G. A. Metzger, W. A. He, and D. C. Guttridge, "Modeling human cancer cachexia in colon 26 tumorbearing adult mice," Journal of Cachexia, Sarcopenia and Muscle, vol. 5, no. 4, pp. 321-328, 2014.

[105] V. A. Lira, M. Okutsu, M. Zhang et al., "Autophagy is required for exercise training-induced skeletal muscle adaptation and improvement of physical performance," FASEB Journal, vol. 27, no. 10, pp. 4184-4193, 2013.

[106] E. J. Jokl and G. Blanco, "Disrupted autophagy undermines skeletal muscle adaptation and integrity," Mammalian Genome, vol. 27, no. 11-12, pp. 525-537, 2016.

[107] N. Kimura, T. Kumamoto, Y. Kawamura et al., "Expression of autophagy-associated genes in skeletal muscle: an experimental model of chloroquine-induced myopathy," Pathobiology, vol. 74, no. 3, pp. 169-176, 2007.

[108] A. S. Nichenko, W. M. Southern, M. Atuan et al., "Mitochondrial maintenance via autophagy contributes to functional skeletal muscle regeneration and remodeling," American Journal of Physiology Cell Physiology, vol. 311, no. 2, pp. C190-C200, 2016.

[109] G. G. Rodney, R. Pal, and R. Abo-Zahrah, "Redox regulation of autophagy in skeletal muscle," Free Radical Biology and Medicine, vol. 98, pp. 103-112, 2016.

[110] M. Sandri, "Autophagy in skeletal muscle," FEBS Letters, vol. 584, no. 7, pp. 1411-1416, 2010.

[111] E. Masiero, L. Agatea, C. Mammucari et al., "Autophagy is required to maintain muscle mass," Cell Metabolism, vol. 10, no. 6, pp. 507-515, 2009.

[112] Y. Luo, J. Yoneda, H. Ohmori et al., "Cancer usurps skeletal muscle as an energy repository," Cancer Research, vol. 74, no. 1, pp. 330-340, 2014.

[113] F. Penna, D. Costamagna, F. Pin et al., "Autophagic degradation contributes to muscle wasting in cancer cachexia," The American Journal of Pathology, vol. 182, no. 4, pp. 13671378, 2013.

[114] L. Tessitore, P. Costelli, G. Bonetti, and F. M. Baccino, "Cancer cachexia, malnutrition, and tissue protein turnover in experimental animals," Archives of Biochemistry and Biophysics, vol. 306, no. 1, pp. 52-58, 1993.

[115] J. R. Mayers, C. Wu, C. B. Clish et al., "Elevation of circulating branched-chain amino acids is an early event in human pancreatic adenocarcinoma development," Nature Medicine, vol. 20, no. 10, pp. 1193-1198, 2014.

[116] U. E. Martinez-Outschoorn, D. Whitaker-Menezes, S. Pavlides et al., "The autophagic tumor stroma model of cancer or "battery-operated tumor growth": a simple solution to the autophagy paradox," Cell Cycle, vol. 9, no. 21, pp. 4297-4306, 2010.

[117] Z. Aversa, F. Pin, S. Lucia et al., "Autophagy is induced in the skeletal muscle of cachectic cancer patients," Scientific Reports, vol. 6, p. 30340, 2016.

[118] F. Penna, S. Busquets, F. Pin et al., "Combined approach to counteract experimental cancer cachexia: eicosapentaenoic acid and training exercise," Journal of Cachexia, Sarcopenia and Muscle, vol. 2, no. 2, pp. 95-104, 2011.

[119] P. F. Cosper and L. A. Leinwand, "Cancer causes cardiac atrophy and autophagy in a sexually dimorphic manner," Cancer Research, vol. 71, no. 5, pp. 1710-1720, 2011.

[120] H. Ding, G. Zhang, K. W. Sin et al., "Activin A induces skeletal muscle catabolism via p38beta mitogen-activated protein kinase," Journal of Cachexia, Sarcopenia and Muscle, vol. 8, no. 2, pp. 202-212, 2017.

[121] A. M. Fritzen, C. Frøsig, J. Jeppesen et al., "Role of AMPK in regulation of LC3 lipidation as a marker of autophagy in skeletal muscle," Cellular Signalling, vol. 28, no. 6, pp. 663-674, 2016.

[122] A. M. Sanchez, A. Csibi, A. Raibon et al., "AMPK promotes skeletal muscle autophagy through activation of forkhead FoxO3a and interaction with Ulk1," Journal of Cellular Biochemistry, vol. 113, no. 2, pp. 695-710, 2012.

[123] P. K. Paul and A. Kumar, "TRAF6 coordinates the activation of autophagy and ubiquitin-proteasome systems in atrophying skeletal muscle," Autophagy, vol. 7, no. 5, pp. 555-556, 2011.

[124] K. Pettersen, S. Andersen, S. Degen et al., "Cancer cachexia associates with a systemic autophagy-inducing activity mimicked by cancer cell-derived IL-6 trans-signaling," Scientific Reports, vol. 7, article 2046, 2017.

[125] K. Baar, "Involvement of PPARg co-activator-1, nuclear respiratory factors 1 and 2, and PPARa in the adaptive response to endurance exercise," Proceedings of the Nutrition Society, vol. 63, no. 2, pp. 269-273, 2004.

[126] P. Puigserver and B. M. Spiegelman, "Peroxisome proliferator-activated receptor-gamma coactivator 1 alpha (PGC-1 alpha): transcriptional coactivator and metabolic regulator," Endocrine Reviews, vol. 24, no. 1, pp. 78-90, 2003.

[127] Z. Wu, P. Puigserver, U. Andersson et al., "Mechanisms controlling mitochondrial biogenesis and respiration through the thermogenic coactivator PGC-1," Cell, vol. 98, no. 1, pp. 115-124, 1999.

[128] F. W. Booth, G. N. Ruegsegger, R. G. Toedebusch, and Z. Yan, "Endurance exercise and the regulation of skeletal muscle metabolism," Progress in Molecular Biology and Translational Science, vol. 135, pp. 129-151, 2015.

[129] P. Zhang, X. Chen, and M. Fan, "Signaling mechanisms involved in disuse muscle atrophy," Medical Hypotheses, vol. 69, no. 2, pp. 310-321, 2007.

[130] S. V. Brooks and J. A. Faulkner, "Contractile properties of skeletal muscles from young, adult and aged mice," The Journal of Physiology, vol. 404, pp. 71-82, 1988.

[131] J. J. Widrick, J. G. Romatowski, K. M. Norenberg et al., "Functional properties of slow and fast gastrocnemius muscle fibers after a 17-day spaceflight," Journal of Applied Physiology, vol. 90, no. 6, pp. 2203-2211, 2001.

[132] A. Garnier, D. Fortin, J. Zoll et al., "Coordinated changes in mitochondrial function and biogenesis in healthy and diseased human skeletal muscle," The FASEB Journal, vol. 19, no. 1, pp. 43-52, 2005.

[133] J. L. Brown, M. E. Rosa-Caldwell, D. E. Lee et al., "PGClalpha4 gene expression is suppressed by the IL-6-MEKERK 1/2 MAPK signalling axis and altered by resistance exercise, obesity and muscle injury," Acta Physiologica (Oxford, England), vol. 220, no. 2, pp. 275-288, 2017. 
[134] J. L. Ruas, J. P. White, R. R. Rao et al., “A PGC-1alpha isoform induced by resistance training regulates skeletal muscle hypertrophy," Cell, vol. 151, no. 6, pp. 1319-1331, 2012.

[135] Z. Arany, N. Lebrasseur, C. Morris et al., "The transcriptional coactivator PGC-1beta drives the formation of oxidative type IIX fibers in skeletal muscle," Cell Metabolism, vol. 5, no. 1, pp. 35-46, 2007.

[136] J. Lin, H. Wu, P. T. Tarr et al., “Transcriptional co-activator PGC-1 alpha drives the formation of slow-twitch muscle fibres," Nature, vol. 418, no. 6899, pp. 797-801, 2002.

[137] A. M. Joseph, H. Pilegaard, A. Litvintsev, L. Leick, and D. A. Hood, "Control of gene expression and mitochondrial biogenesis in the muscular adaptation to endurance exercise," Essays in Biochemistry, vol. 42, pp. 13-29, 2006.

[138] V. A. Lira, C. R. Benton, Z. Yan, and A. Bonen, "PGC-1alpha regulation by exercise training and its influences on muscle function and insulin sensitivity," American Journal of Physiology Endocrinology and Metabolism, vol. 299, no. 2, pp. E145-E161, 2010.

[139] A. Vainshtein, L. D. Tryon, M. Pauly, and D. A. Hood, "Role of PGC-1alpha during acute exercise-induced autophagy and mitophagy in skeletal muscle," American Journal of Physiology Cell Physiology, vol. 308, no. 9, pp. C710-9, 2015.

[140] J. Cannavino, L. Brocca, M. Sandri, R. Bottinelli, and M. A. Pellegrino, "PGC1-alpha over-expression prevents metabolic alterations and soleus muscle atrophy in hindlimb unloaded mice," The Journal of Physiology, vol. 592, no. 20, pp. 45754589, 2014.

[141] J. Cannavino, L. Brocca, M. Sandri, B. Grassi, R. Bottinelli, and M. A. Pellegrino, "The role of alterations in mitochondrial dynamics and PGC-1alpha over-expression in fast muscle atrophy following hindlimb unloading," The Journal of Physiology, vol. 593, no. 8, pp. 1981-1995, 2015.

[142] A. Vainshtein, E. M. Desjardins, A. Armani, M. Sandri, and D. A. Hood, "PGC-1alpha modulates denervation-induced mitophagy in skeletal muscle," Skeletal Muscle, vol. 5, p. 9, 2015.

[143] M. Sandri, J. Lin, C. Handschin et al., "PGC-1alpha protects skeletal muscle from atrophy by suppressing FoxO3 action and atrophy-specific gene transcription," Proceedings of the National Academy of Sciences of the United States of America, vol. 103, no. 44, pp. 16260-16265, 2006.

[144] M. J. Puppa, E. A. Murphy, R. Fayad, G. A. Hand, and J. A. Carson, "Cachectic skeletal muscle response to a novel bout of low-frequency stimulation," Journal of Applied Physiology, vol. 116, no. 8, pp. 1078-1087, 2014.

[145] X. Wang, A. M. Pickrell, T. A. Zimmers, and C. T. Moraes, "Increase in muscle mitochondrial biogenesis does not prevent muscle loss but increased tumor size in a mouse model of acute cancer-induced cachexia," PloS One, vol. 7, no. 3, article e33426, 2012.

[146] S. Jager, C. Handschin, J. St-Pierre, and B. M. Spiegelman, "AMP-activated protein kinase (AMPK) action in skeletal muscle via direct phosphorylation of PGC-1alpha," Proceedings of the National Academy of Sciences of the United States of America, vol. 104, no. 29, pp. 1201712022, 2007.

[147] R. Bergeron, J. M. Ren, K. S. Cadman et al., "Chronic activation of AMP kinase results in NRF-1 activation and mitochondrial biogenesis," American Journal of Physiology Endocrinology and Metabolism, vol. 281, no. 6, pp. E1340-6, 2001.
[148] M. M. Mihaylova and R. J. Shaw, “The AMPK signalling pathway coordinates cell growth, autophagy and metabolism," Nature Cell Biology, vol. 13, no. 9, pp. 1016-1023, 2011.

[149] D. Carling, "AMPK signalling in health and disease," Current Opinion in Cell Biology, vol. 45, pp. 31-37, 2017.

[150] B. Cool, B. Zinker, W. Chiou et al., "Identification and characterization of a small molecule AMPK activator that treats key components of type 2 diabetes and the metabolic syndrome," Cell Metabolism, vol. 3, no. 6, pp. 403-416, 2006.

[151] J. P. White, M. J. Puppa, S. Gao, S. Sato, S. L. Welle, and J. A. Carson, "Muscle mTORC1 suppression by IL-6 during cancer cachexia: a role for AMPK," American Journal of Physiology Endocrinology and Metabolism, vol. 304, no. 10, pp. E1042-E1052, 2013.

[152] X. Ge, A. Vajjala, C. McFarlane, W. Wahli, M. Sharma, and R. Kambadur, "Lack of Smad3 signaling leads to impaired skeletal muscle regeneration," American Journal of Physiology Endocrinology and Metabolism, vol. 303, no. 1, pp. E90-102, 2012.

[153] S. M. Hindi, V. Mishra, S. Bhatnagar et al., "Regulatory circuitry of TWEAK-Fn14 system and PGC-1alpha in skeletal muscle atrophy program," The FASEB Journal, vol. 28, no. 3, pp. 1398-1411, 2014.

[154] A. J. Grande, V. Silva, R. Riera et al., "Exercise for cancer cachexia in adults," Cochrane Database of Systematic Reviews, vol. 11, article CD010804, 2014.

[155] F. W. Booth, C. K. Roberts, and M. J. Laye, "Lack of exercise is a major cause of chronic diseases," Comprehensive Physiology, vol. 2, no. 2, pp. 1143-1211, 2012.

[156] J. O. Holloszy and E. F. Coyle, “Adaptations of skeletal muscle to endurance exercise and their metabolic consequences," Journal of Applied Physiology: Respiratory, Environmental and Exercise Physiology, vol. 56, no. 4, pp. 831-838, 1984.

[157] J. O. Holloszy, “Adaptations of skeletal muscle mitochondria to endurance exercise: a personal perspective," Exercise and Sport Sciences Reviews, vol. 32, no. 2, pp. 41-43, 2004.

[158] O. Holloszy, "Enzymatic adaptations of skeletal muscle to endurance exercise," Current Problems in Clinical Biochemistry, vol. 11, pp. 118-121, 1982.

[159] D. A. Hood, "Invited review: contractile activity-induced mitochondrial biogenesis in skeletal muscle," Journal of Applied Physiology, vol. 90, no. 3, pp. 1137-1157, 2001.

[160] S. al-MajidD. O. McCarthy, "Resistance exercise training attenuates wasting of the extensor digitorum longus muscle in mice bearing the colon-26 adenocarcinoma," Biological Research for Nursing, vol. 2, no. 3, pp. 155-166, 2001.

[161] D. C. Wright, D. H. Han, P. M. Garcia-Roves, P. C. Geiger, T. E. Jones, and J. O. Holloszy, "Exercise-induced mitochondrial biogenesis begins before the increase in muscle PGClalpha expression," The Journal of Biological Chemistry, vol. 282, no. 1, pp. 194-199, 2007.

[162] G. D. Stewart, R. J. Skipworth, and K. C. Fearon, "Cancer cachexia and fatigue," Clinical Medicine (London, England), vol. 6, no. 2, pp. 140-143, 2006.

[163] E. Ferriolli, R. J. Skipworth, P. Hendry et al., "Physical activity monitoring: a responsive and meaningful patient-centered outcome for surgery, chemotherapy, or radiotherapy?," Journal of Pain and Symptom Management, vol. 43, no. 6, pp. 1025-1035, 2012. 
[164] J. Farkas, S. Haehlingvon, K. Kalantar-Zadeh, J. E. Morley, S. D. Anker, and M. Lainscak, "Cachexia as a major public health problem: frequent, costly, and deadly," Journal of Cachexia, Sarcopenia and Muscle, vol. 4, pp. 173-178, 2013.

[165] A. Montazeri, "Quality of life data as prognostic indicators of survival in cancer patients: an overview of the literature from 1982 to 2008," Health and Quality of Life Outcomes, vol. 7, p. 102, 2009.

[166] K. A. Baltgalvis, F. G. Berger, M. M. Peña, J. Mark Davis, J. P. White, and J. A. Carson, "Activity level, apoptosis, and development of cachexia in $A p c^{\mathrm{Min} /+}$ mice," Journal of Applied Physiology, vol. 109, no. 4, pp. 1155-1161, 2010.

[167] K. T. Murphy, A. Chee, J. Trieu, T. Naim, and G. S. Lynch, "Importance of functional and metabolic impairments in the characterization of the C-26 murine model of cancer cachexia," Disease Models \& Mechanisms, vol. 5, no. 4, pp. 533-545, 2012.

[168] F. W. Booth and M. V. Chakravarthy, "Physical activity and dietary intervention for chronic diseases: a quick fix after all?" Journal of Applied Physiology, vol. 100, no. 5, pp. 14391440, 2006.

[169] M. Dahele, R. J. Skipworth, L. Wall, A. Voss, T. Preston, and K. C. Fearon, "Objective physical activity and self-reported quality of life in patients receiving palliative chemotherapy," Journal of Pain and Symptom Management, vol. 33, no. 6, pp. 676-685, 2007.

[170] A. Gratas-Delamarche, F. Derbré, S. Vincent, and J. Cillard, "Physical inactivity, insulin resistance, and the oxidativeinflammatory loop," Free Radical Research, vol. 48, no. 1, pp. 93-108, 2014.

[171] J. P. Thyfault and F. W. Booth, "Lack of regular physical exercise or too much inactivity," Current Opinion in Clinical Nutrition and Metabolic Care, vol. 14, no. 4, pp. 374-378, 2011.

[172] M. J. Puppa, J. P. White, K. T. Velázquez et al., “The effect of exercise on IL-6-induced cachexia in the Apc (Min/+) mouse," Journal of Cachexia, Sarcopenia and Muscle, vol. 3, no. 2, pp. 117-137, 2012.

[173] J. P. Hardee, J. E. Mangum, S. Gao et al., "Eccentric contraction-induced myofiber growth in tumor-bearing mice," Journal of Applied Physiology, vol. 120, no. 1, pp. 2937, 2016.

[174] A. Sheldon, F. W. Booth, and C. R. Kirby, "cAMP levels in fast- and slow-twitch skeletal muscle after an acute bout of aerobic exercise," The American Journal of Physiology, vol. 264, 6 Part 1, pp. C1500-C1504, 1993.

[175] A. M. Sanchez, "Autophagy regulation in human skeletal muscle during exercise," The Journal of Physiology, vol. 594, no. 18, pp. 5053-5054, 2016.

[176] A. M. Sanchez, H. Bernardi, G. Py, and R. B. Candau, "Autophagy is essential to support skeletal muscle plasticity in response to endurance exercise," American Journal of Physiology. Regulatory, Integrative and Comparative Physiology, vol. 307, no. 8, pp. R956-R969, 2014.

[177] C. Schwalm, C. Jamart, N. Benoit et al., "Activation of autophagy in human skeletal muscle is dependent on exercise intensity and AMPK activation," The FASEB Journal, vol. 29, no. 8, pp. 3515-3526, 2015.
[178] A. Vainshtein, P. Grumati, M. Sandri, and P. Bonaldo, "Skeletal muscle, autophagy, and physical activity: the menage a trois of metabolic regulation in health and disease," Journal of Molecular Medicine (Berlin, Germany), vol. 92, no. 2, pp. 127-137, 2014.

[179] E. Pigna, E. Berardi, P. Aulino et al., "Aerobic exercise and pharmacological treatments counteract cachexia by modulating autophagy in colon cancer," Scientific Reports, vol. 6, article 26991, 2016. 


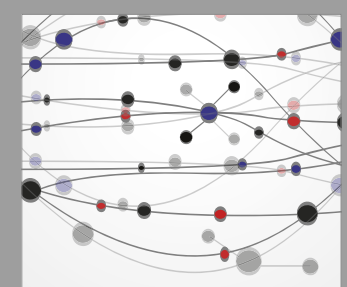

The Scientific World Journal
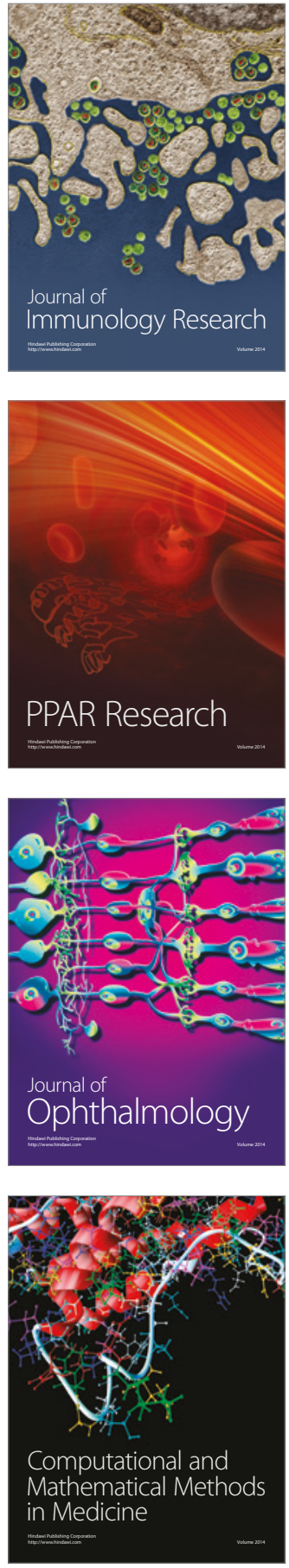

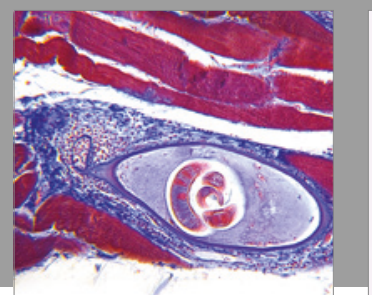

Gastroenterology Research and Practice
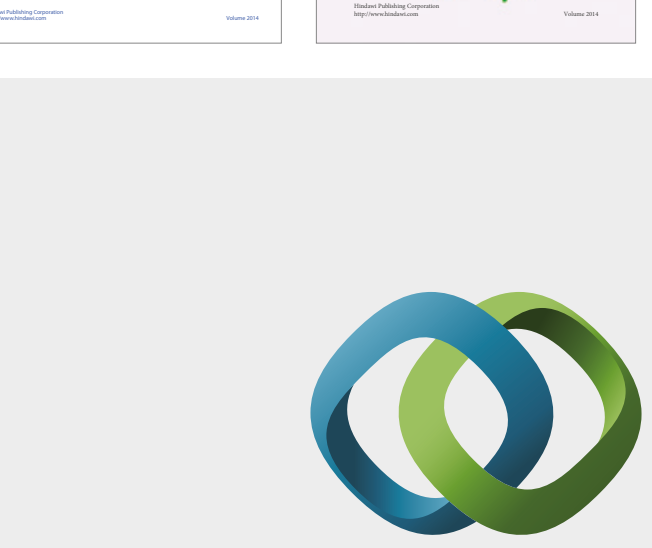

\section{Hindawi}

Submit your manuscripts at

https://www.hindawi.com
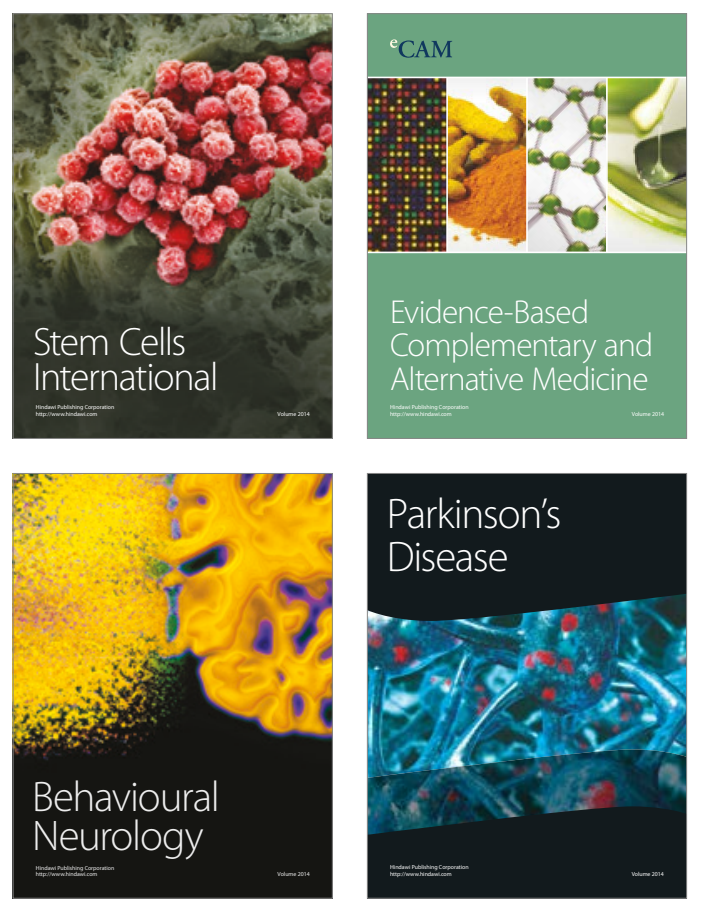
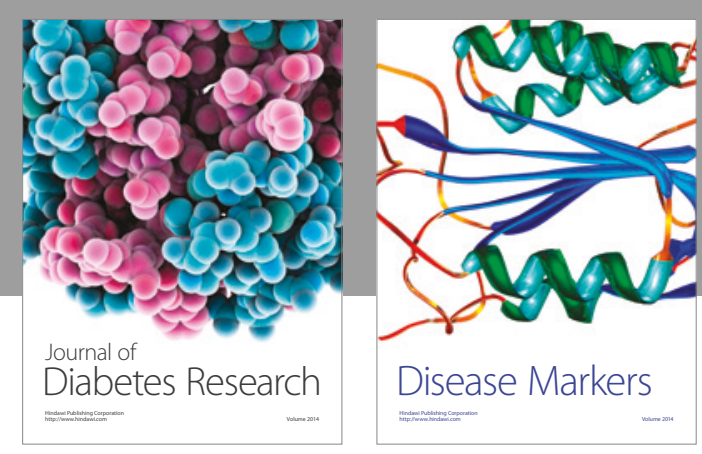

Disease Markers
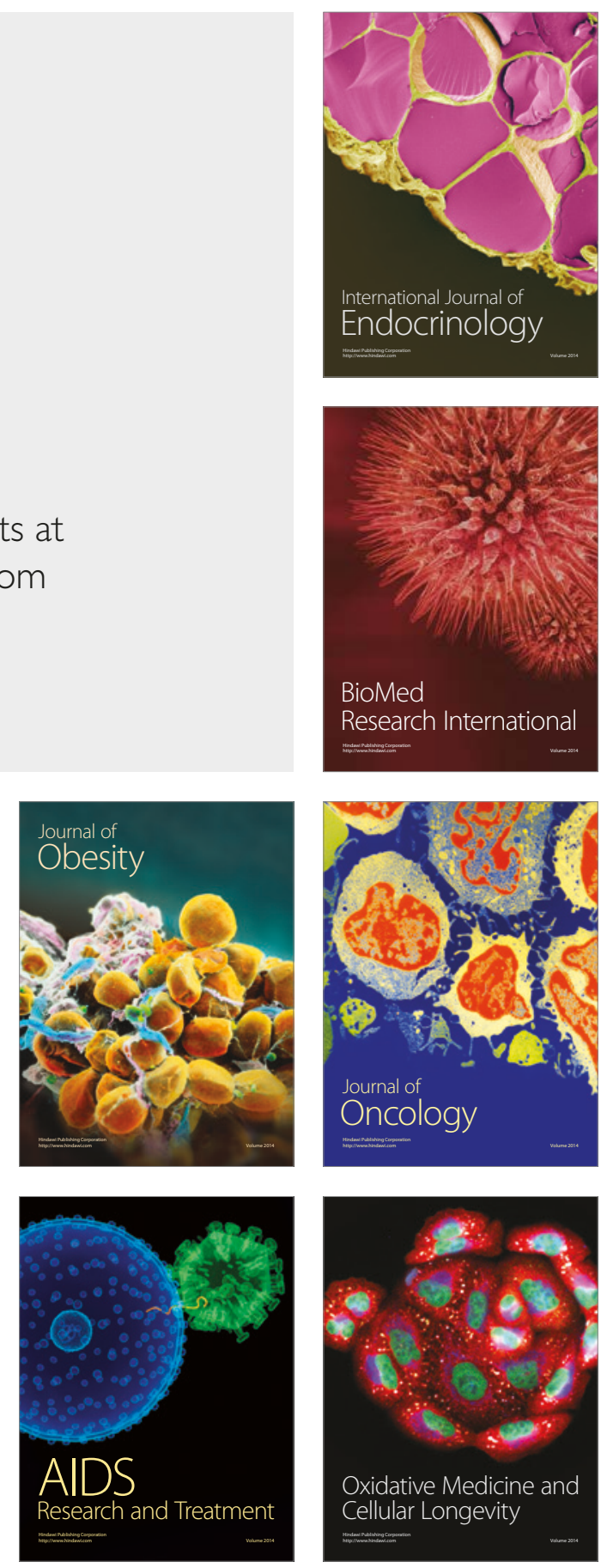